\title{
Síntomas depresivos en adultos mayores institucionalizados y factores asociados*
}

\author{
Depressive Symptoms in Institutionalized \\ Elderly People and Factors Associated
}

Recibido: noviembre 20 de 2010 | Revisado: diciembre 11 de 2011 | Aceptado: marzo 19 de 2012

\author{
Alejandro Estrada Restrepo ** \\ Universidad de Antioquia, Medellín, Colombia \\ DORIS CARDONA ARANGO *** \\ Ángela María Segura CARDONa \\ JAIME ORDÓÑEZ MOLINA \\ JORGE JuLiÁN OSORIO GÓMEZ \\ Universidad CES, Medellín, Colombia \\ LiNa Marcela Chavarriaga MaYA ***** \\ Universidad Pontificia Bolivariana, Medellín, Colombia
}

\section{RESUMEN}

Este estudio tuvo como objetivo explorar los factores asociados con síntomas depresivos en adultos mayores institucionalizados. Se realizó un estudio transversal en 276 adultos mayores de Centros de Bienestar del Anciano de Medellín. Se valoraron características demográficas, funcionales, ansiedad, salud percibida, redes de apoyo, estado nutricional y calidad de vida. Prevalencias de sintomatología depresiva fueron similares por sexo y edad $(p>0.05)$. Adultos dependientes para la realización de actividades cotidianas, tuvieron mayores porcentajes de síntomas de depresión $(p<0.001)$. Altos niveles de ansiedad $(\mathrm{OR}=2.74)$, deterioro funcional $(\mathrm{OR}=2.82)$, ningún grado de formación académica $(\mathrm{OR}=3.70)$ y mujeres parcialmente dependientes $(\mathrm{OR}=21.89)$ se asociaron con mayor probabilidad de presentar síntomas de depresión $(p<0.05)$. En general, perdida de roles, estado de dependencia y calidad de vida disminuida aumentan la sintomatología depresiva.

Para citar este artículo: Estrada, A., Cardona, D., Segura, A. M., Ordóñez, J., Osorio, J. J. \& Chavarriaga, L. M. (2013). Síntomas depresivos en adultos mayores institucionalizados y factores asociados. Universitas Psychologica, 12(1), 81-94.

* Agradecimientos: Los investigadores agradecen a los Centros de Bienestar del Anciano y a los adultos mayores por participar en este proceso. De igual manera, a la Dirección de Investigación de la Universidad CES, Medellín-Colombia, por la financiación del proyecto.

*** Escuela de Nutrición y Dietética - Universidad de Antioquia. Carrera 75 № 65-87 Medellín, (57-4) 21992 20, 2199200 Fax (57 4) 23050 07. E-mail: aestrada@pijaos.udea.edu.co

**** Universidad CES. Calle 10 A No. 22 - 04 Medellín, (57-4) 44405 55. E-mails: dcardona@ces.edu.co, asegura@ces.edu.co, jordonez@ces.edu.co, josorio@ces.edu.co

****** Universidad Pontificia Bolivariana. Calle 78 B \# 72 A 109 Medellín, (57-4) 49363 00. E-mail: linachm@yahoo.com

Palabras clave autores

Depresión, adulto mayor, calidad de vida, institucionalización, Medellín.

Palabras clave descriptores

Psicología de la salud, Psicología clínica, Colombia

\section{A B S T R A C T}

The objective of the present study was exploring the factors associated with depressive symptoms in elderly people institutionalized. A cross sectional study was conducted; 276 elderly people of elderly welfare centers of Medellin-Colombia were analyzed. Demographics, capacity functional, anxiety, perceived health, social support networks, nutritional status and quality of life characteristics were evaluated. The depressive symptoms prevalence rate in the subjects was similar by sex an age $(p>0.05)$. The dependent elderly performing the activities of daily living had the upper percentages of depressive symptoms $(p<0.001)$. Risk of anxiety $(\mathrm{OR}=2.74)$, cognitive impairment $(\mathrm{OR}=2.82)$, iliteracy and women partially dependent $(\mathrm{OR}=21.89)$ increased significantly the probability of depressive symptoms $(p<0.05)$. Therefore, the loss of roles, dependency status and diminishes quality of life increase the depressive symptomatology.

Key words authors

Depression, elderly, quality of life, institutionalization, Medellin.

Key words plus

Health Psychology, Clinical Psychology, Colombia. 


\section{Introducción}

La depresión es un trastorno que se ha convertido en un importante problema de salud pública a nivel mundial, el cual es considerado uno de los principales problemas de salud mental de los adultos mayores (Conde \& Jarne, 2006; Lebowitz et al., 1997). Esta lleva consigo condiciones como aislamiento social, baja productividad, discapacidades, postramiento, sobremedicación (Alarcón \& García, 2003; Gómez-Restrepo et al., 2004; Urbina, Flores, García, Torres \& Torrubias, 2007), circunstancias que potencian la aparición de este tipo de trastornos depresivos. Además, se presenta la subvaloración que se da por parte del personal asistencial al diagnóstico de esta patología, asumiendo que es un proceso normal del envejecimiento, desconociendo que la depresión está estrechamente relacionada con la calidad de vida del adulto mayor (Alexandre, Cordeiro \& Ramos, 2009; Chachamovich, Fleck, Laidlaw \& Power, 2008; Wada et al., 2005).

Aunado a esto, otra de las situaciones problematizadoras para los adultos mayores es su rápido crecimiento como grupo poblacional a nivel mundial, pero de especial forma en las regiones en vías de desarrollo; es así como las Naciones Unidas (2009) proyectan para Latinoamérica que el 9\% del total de su población en el 2020 serán personas con 65 años o más, y para países como Brasil, Argentina, Chile y Uruguay estas cifras serán 9.6\%, $11.9 \%, 12.1 \%$ y $15 \%$, respectivamente; mientras que para Ecuador y Bolivia serán $8.8 \%$ y $5.8 \%$. Por su parte y según datos del censo de Colombia del 2005, el 6.3\% de la población tenía 65 ó más años y se proyecta que para el 2020 esta cifra sea del orden de $8.5 \%$ (Departamento Administrativo Nacional de Estadística, 2007).

A su vez, los adultos mayores concentran el grupo con más limitaciones funcionales que afectan su independencia en el diario vivir, por lo que requieren una valoración geriátrica integral y asistencia especial; además, se incrementa el número de individuos con trastornos mentales, cognitivos y del comportamiento, lo que hace más difícil su cuidado a largo plazo (Hirschfeld \& Lindsey, 2002). En el caso específico para Colom- bia este contexto plantea la necesidad de mayores coberturas del sistema de seguridad social en salud, así como un mayor número de instituciones geriátricas y/o mejoría de las existentes, con el fin de poder asistir de una manera adecuada a este grupo poblacional.

Estas entidades han llevado un proceso de institucionalización del anciano que ha tenido como una de sus principales finalidades resolver los problemas derivados de los escasos o nulos recursos económicos, de la falta de apoyo, del abandono, de la marginación social, etc., en el que se encuentra el adulto mayor. Es sabido que el mayor consumo de recursos sanitarios y sociales acontece en esta etapa de la vida, y que la relación que toda persona tenga con el anciano será de gran ayuda para su integración con la familia y la sociedad (Aranda, Pando, Flores \& García, 2001).

De forma adicional, se conoce que dentro de este proceso de institucionalización surgen algunas características negativas de las organizaciones de internación como son el cambio de contexto para el adulto mayor, dejando de obtener reconocimiento por parte de los vecinos, familiares y amigos; el sentimiento de carga e inutilidad; el desarraigo, generando expectativas básicas que no son colmadas; el aislamiento con el medio, el maltrato, entre otras (Cerquera, 2008; Minor \& Kaemppffmam, 2006).

Asimismo, se ha reportado que en los adultos mayores que viven en residencias, las condiciones de vida y la falta de contacto social se han asociado con síntomas depresivos (Santos et al., 2010). Además, los estudios de Pérez y Arcia (2008) han reportado asociación entre viudez con una mayor probabilidad de síntomas depresivos, al igual que el estado de salud física, el desarrollo de actividades de la vida diaria y la autonomía de los adultos mayores.

Por lo expuesto, este estudio explora la asociación de factores como salud física y mental, condiciones de apoyo social, estado nutricional y calidad de vida con los síntomas de depresión en la población adulta mayor institucionalizada en los Centros de Bienestar del Anciano (CBA) de la ciudad de Medellín (Colombia) en el 2008. 


\section{Métodos}

\section{Tipo de estudio}

Se diseñó un estudio de tipo descriptivo transversal, que tuvo como población de referencia las personas de 65 años y más que vivían en los 103 CBA de la zona urbana registrados en la Secretaría de Bienestar Social del municipio de Medellín (Colombia) al año 2008.

\section{Participantes}

El tamaño de la muestra estuvo condicionado a la autorización para participar en 39 centros, los demás centros no permitieron el ingreso, por razones como que su población objetivo no eran personas mayores de 65 años o más, o asistían adultos mayores con problemas mentales graves. Finalmente, el estudio evaluó 276 ancianos, de los cuales el 40.6\% eran hombres con una edad promedio $77.6 \pm 8.4$ años, y el 59.4\% mujeres con edad promedio $79.5 \pm$ 7.7 años. Fueron excluidos los adultos mayores con deterioro cognitivo que les impidiera responder el cuestionario y aquellos que no tenían la institución como residencia permanente.

\section{Instrumentos}

La información recopilada relacionó aspectos demográficos, de apoyo social, condiciones de salud percibida, capacidad funcional, satisfacción personal, nivel de depresión, nivel de ansiedad, mini evaluación nutricional y calidad de vida.

Para estimar los síntomas depresivos se utilizó la escala de valoración geriátrica de depresión de Yesavage en su versión reducida de 15 ítems -respuestas dicotómicas- (Yesavage et al., 1982). Se utilizó un punto de corte de 6 o más para considerar presencia de síntomas depresivos. Estudios realizados en Colombia han mostrado una consistencia interna del 0.727 para esta escala (Bacca, González $\&$ Uribe, 2005). Para el nivel de ansiedad se usó la escala de ansiedad de Goldberg (Goldberg, Bridges, Duncan-Jones \& Grayson, 1988), que consta de nueve preguntas -respuestas dicotómicas-, pero las cinco últimas, solo se formulaban si hay respuestas positivas en dos o más de las cuatro primeras preguntas, las cuales son obligatorias. El punto de corte para ansiedad se planteó en cuatro o más.

Para la estimación del estado nutricional se usó el cribado de la minivaloración nutricional (MNA, por sus siglas en inglés). Se utilizó la versión corta de la escala con un puntaje máximo de 14 puntos, donde 11 puntos o menos clasifican al adulto mayor como en posible malnutrición y 12 o más en estado nutricional normal (Guigoz, Vellas \& Garry, 1994).

Para la valoración de la calidad de vida, se utilizó la escala de calidad de vida en adultos mayores -WHOQOL-OLD de la Organización Mundial de la Salud-. Esta consta de 24 ítems tipo Likert; el puntaje se transforma en una valoración que va de 0 a 100, donde puntajes altos indican una alta calidad de vida (Organización Mundial de la Salud, 2006). Para la evaluación funcional del adulto mayor se utilizó la escala Pfeffer, la cual es usada para registrar el deterioro funcional temprano, detectando actividades que se ven disminuidas por problemas cognoscitivos (Ávila-Funes, Garant \& Aguilar-Navarro, 2006); puntajes mayores o iguales a seis, sugieren déficit funcional en el adulto mayor.

Los demás aspectos demográficos, autonomía (dependiente, parcialmente dependiente, independiente), condiciones de salud diagnosticadas por personal médico y autopercibidas, condición de estadía en la institución (caridad, pensionado), motivos de la institucionalización, maltrato por parte de los cuidadores o los familiares, satisfacción de los adultos mayores con el apoyo recibido por parte de amigos y familiares (calificado de 1 a 5 , siendo 1 altamente insatisfecho y 5 altamente satisfecho), entre otras, se captaron por medio de una encuesta diseñada por los investigadores.

\section{Procedimiento}

Una prueba piloto fue llevada a cabo con el fin de evitar redundancias en la información, garantizar que se recopilaran todos los datos y ajustar la encuesta; de igual manera, los encuestadores, fueron estandarizados y capacitados para la recolección de toda la información. Además, la toma de medidas 
antropométricas requeridas para el cribado del MNA, como peso y talla, se realizó según los estándares de medición de uso internacional (Jackson \& Pollock, 1985). La toma de los datos de los adultos mayores se realizó en el mismo CBA donde vivía el adulto, buscando siempre que el sujeto estuviese cómodo y sin la cercanía de otros adultos o de los cuidadores.

De forma adicional, una cláusula de confidencialidad fue incluida en los formularios, solicitando también consentimiento informado escrito por parte de los adultos mayores o del representante legal de la institución para la realización de la encuesta. Durante el proyecto se acogieron las consideraciones éticas planteadas en la Resolución 8430 de 1993 del Ministerio de Salud de Colombia que reglamenta la investigación en seres vivos. El estudio fue aprobado por el Comité de Ética de la Universidad CES.

\section{Análisis de datos}

Los datos fueron descritos mediante porcentajes, promedio y desviación estándar. La asociación entre las variables estudiadas se efectuó a partir de la prueba chi cuadrado y la prueba $U$ de Mann Whitney; se utilizó un nivel de significación estadística del 5\%. Se estimaron las razones de disparidad (OR) y sus intervalos de confianza del 95\% para determinar los factores asociados a síntomas depresivos, los cuales fueron ajustados por medio de una regresión logística. Este análisis se llevó a cabo en el programa SPSS 17.0. De igual manera, se efectuó un análisis de clases latentes (Agresti, 2002), para ello, se tuvo en cuenta las variables edad, sexo, nivel educativo, sintomatología depresiva, soledad como motivo para institucionalizarse, si la familia lo llevó a la institución, institucionalización voluntaria, satisfacción con el apoyo recibido por los familiares,

TABLA 1

Nivel de depresión según características sociodemográficas y condición del adulto mayor institucionalizado

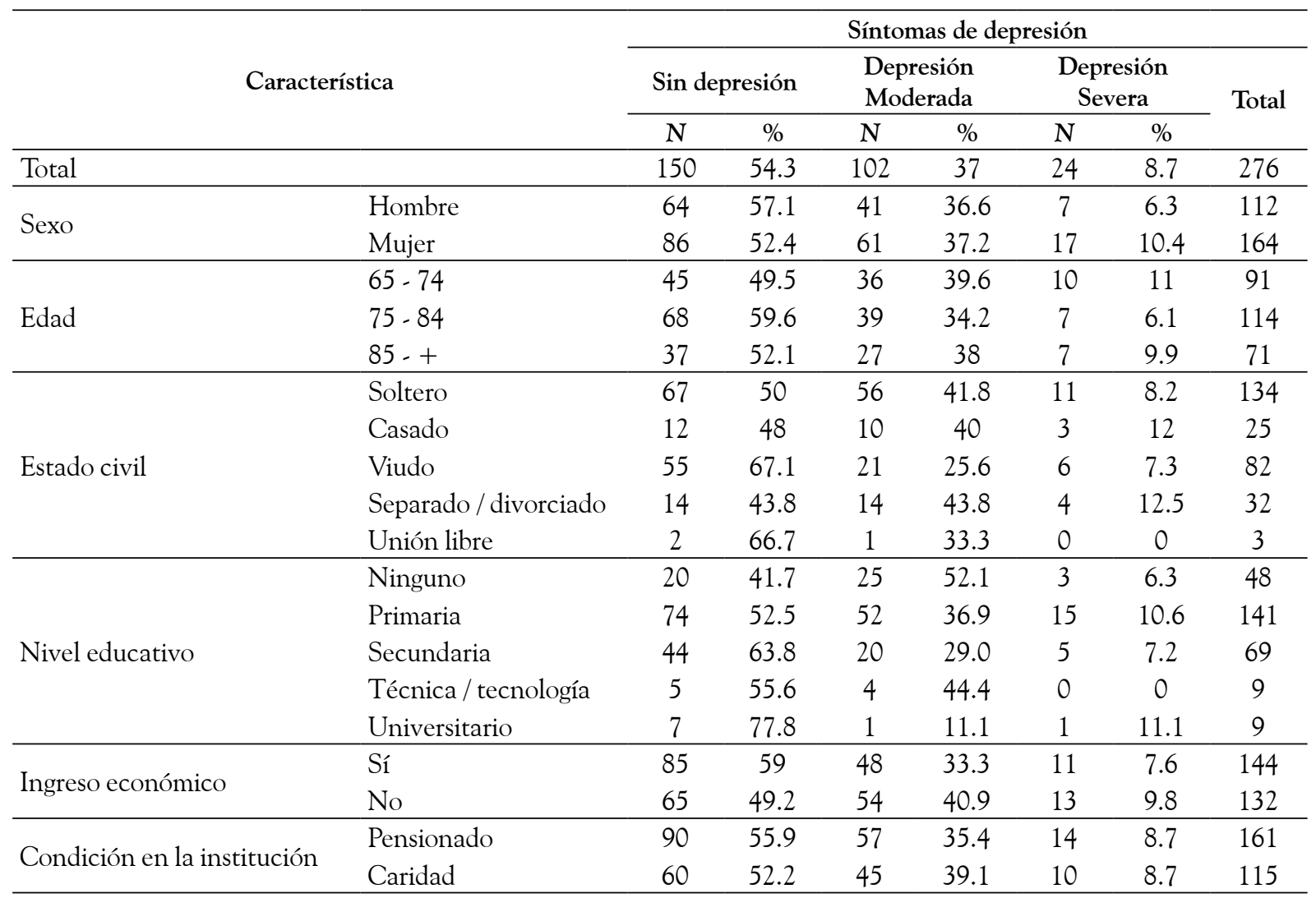

Fuente: elaboración propia. 
amigos e institución, presencia de problemas de diabetes, consideración del estado de salud, autonomía para la realización de actividades cotidianas, clasificación del estado nutricional y percepción de la calidad de vida, las cuales se analizaron en el software R versión 2.9.0. Para determinar el número óptimo de clases latentes y el ajuste del modelo, se calculó el criterio bayesiano de clasificación (BIC) y el estadístico de verosimilitud. El modelo que presentó el menor BIC, fue el modelo que mejor ajustó a las clases.

\section{Resultados}

En la población adulta mayor evaluada en los CBA, se encontró que $41.3 \%$ tenía edades entre los 75 y 84 años, seguido por 33\% de adultos mayores con edades entre 65 y 74 años, mientras que 25.7\% tenía 85 o más años; solo se hallaron dos personas con edades mayores de 100 años. El nivel educativo que predominó fue la primaria $(51.1 \%)$, seguida por la secundaria $(25 \%)$, se halló que $17.4 \%$ no tenían ningún nivel de formación. En cuanto al Sistema de Seguridad Social en Salud, se hallaba afiliado a este el 93.1\%; de estos, se encontraban afiliados al régimen contributivo $51 \%$, mientras que $46.3 \%$ lo estaba en el régimen subsidiado. Además 58.3\% de los adultos mayores estaban en la institución como pensionados.

De acuerdo al puntaje obtenido en la escala de síntomas depresivos, se halló que 54.3\% (150) de los adultos mayores institucionalizados encuestados no registraron ningún grado de síntomas de depresión; presentó síntomas depresivos moderados el 37\% (102) y el $8.7 \%$ (24) los registraron de forma severa.

La prevalencia de síntomas depresivos fue $47.6 \%$ en las mujeres, similar a los hombres $(p=0.4398)$. Por edad, el porcentaje más alto de adultos mayores sin depresión se halló en los de 75 a 84 años (59.6\%), similar a los otros grupos de edad ( $p=0.3095)$. Por otro lado, las personas que no presentaron ningún nivel educativo tuvieron la mayor prevalencia de depresión (58.4\%) $(p=0.0820)$ (Tabla 1).

Los adultos que manifestaron la soledad como motivo para la institucionalización, mostraron una prevalencia de síntomas depresivos similar a los que no se sentían solos $(p=0.9821)$. Asimismo, los problemas de salud y económicos no se hallaron asociados con síntomas de depresión $(p>0.05)$

En los adultos que la familia los llevó a la institución geriátrica, la proporción de síntomas depresivos no mostró diferencias significativas con aquellos que no fueron llevados por sus familiares $(p=0.1513)$. Los adultos que manifestaron haber sido maltratados por familiares, mostraron mayor sintomatología depresiva $(p=0.0153)$ (Tabla 2$)$.

De acuerdo a las condiciones de salud percibida, los adultos hipertensos tuvieron un menor porcentaje de síntomas de depresión ( $p=0.1996)$. Situación similar se halló en los adultos que manifestaron tener problemas del corazón $(p=0.2131)$ y en los huesos $(p=0.1442)$. Por su parte, en los adultos que padecían problemas nerviosos $(p=0.0012)$ y los que eran dependientes para realizar sus actividades cotidianas $(p<0.0001)$, la prevalencia de síntomas depresivos fue superior; al igual que en los que habían sufrido estrés o una enfermedad aguda $(p=0.0001)$, y los que presentaban posible malnutrición ( $p=0.0368$ ) (Tabla 3).

El hecho de tener riesgo de ansiedad mostró prevalencias más elevadas de síntomas de depresión moderada (54.9\%) y severa (23.1\%), en comparación con los adultos sin este riesgo (28.1\% síntomas de depresión moderada; $1.6 \%$ severa) $(p<0.0001)$. Las actividades funcionales se asociaron con la presencia de depresión $(p<0.05)$, siendo los adultos con déficit funcional los que tuvieron mayores prevalencias de síntomas depresivos (53.8\% síntomas moderados y $16.7 \%$ síntomas severos), en comparación con los de una adecuada funcionalidad $30.3 \%$ (moderados) y $5.6 \%$ (severos).

De igual manera, se halló una diferencia entre el nivel de depresión y el puntaje de calidad de vida $(p<0.0001)$, encontrando como el puntaje medio de calidad de vida fue más bajo en aquellos adultos mayores con síntomas depresivos severos (39.8 \pm 10$)$, en comparación con los moderados $(52.9 \pm 11.3)$ y los que no tuvieron síntomas de depresión $(62.6 \pm 10.1)$.

Después de aplicar un modelo de regresión logística para estimar el riesgo de que un adulto mayor institucionalizado presentara síntomas depresivos, 
TABLA 2

Nivel de depresión según características relacionadas con el apoyo social del adulto mayor institucionalizado

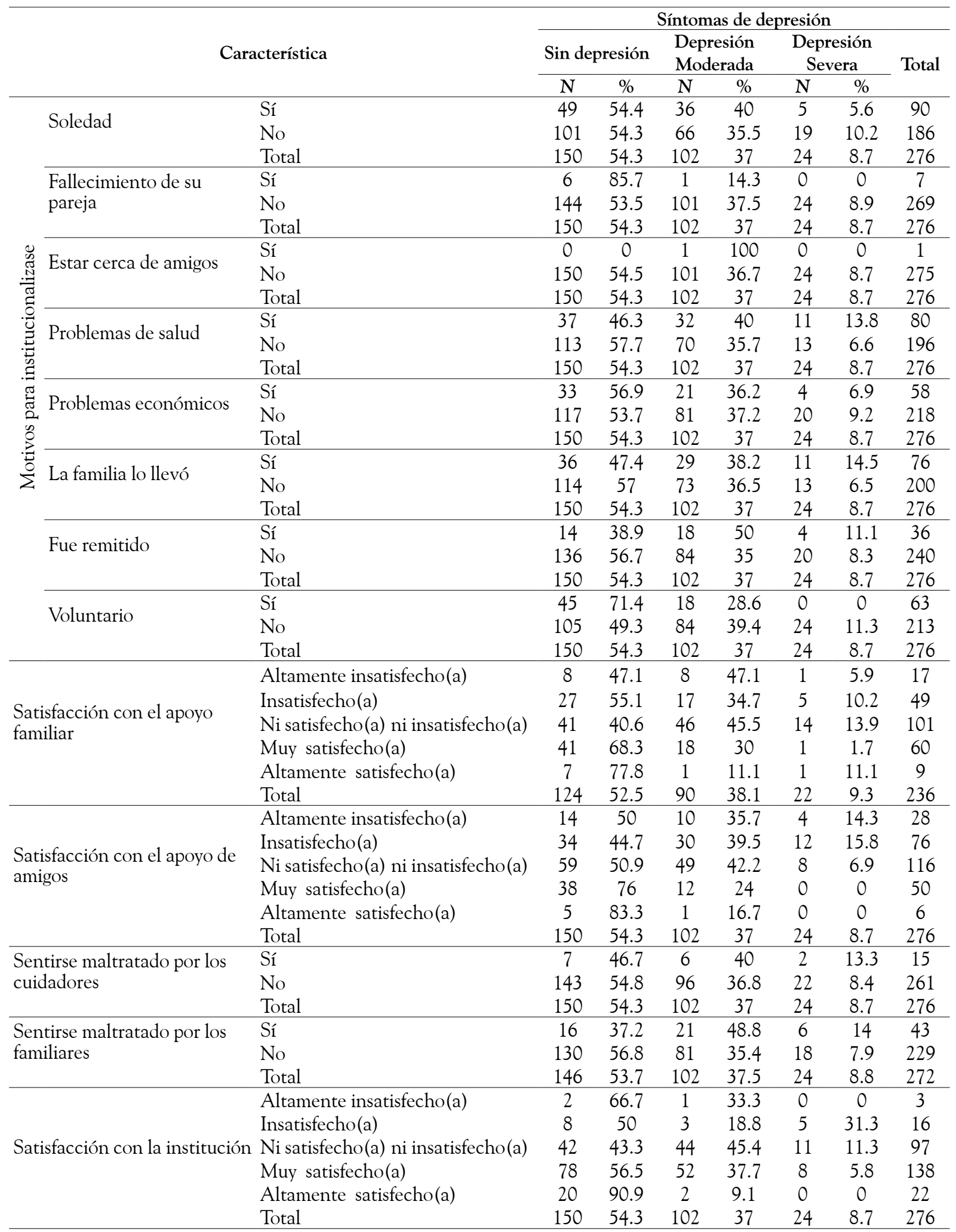

Fuente: elaboración propia. 
TABLA 3

Nivel de depresión según condición de salud percibida del adulto mayor institucionalizado

\begin{tabular}{|c|c|c|c|c|c|c|c|c|}
\hline \multirow{3}{*}{ Característica } & & \multicolumn{7}{|c|}{ Síntomas de depresión } \\
\hline & & \multicolumn{2}{|c|}{$\begin{array}{c}\text { Sin } \\
\text { depresión }\end{array}$} & \multicolumn{2}{|c|}{$\begin{array}{l}\text { Depresión } \\
\text { Moderada }\end{array}$} & \multicolumn{2}{|c|}{$\begin{array}{c}\text { Depresión } \\
\text { Severa }\end{array}$} & \multirow[t]{2}{*}{ Tota } \\
\hline & & $\mathrm{N}$ & $\%$ & $\mathrm{~N}$ & $\%$ & $\mathrm{~N}$ & $\%$ & \\
\hline \multirow{3}{*}{ Hipertensión Arterial } & Sí & 86 & 56.2 & 54 & 35.3 & 13 & 8.5 & 153 \\
\hline & No & 49 & 48 & 42 & 41.2 & 11 & 10.8 & 102 \\
\hline & Total & 135 & 52.9 & 96 & 37.6 & 24 & 9.4 & 255 \\
\hline \multirow{3}{*}{ Diabetes } & Sí & 26 & 55.3 & 15 & 31.9 & 6 & 12.8 & 47 \\
\hline & No & 109 & 52.4 & 81 & 38.9 & 18 & 8.7 & 208 \\
\hline & Total & 135 & 52.9 & 96 & 37.6 & 24 & 9.4 & 255 \\
\hline \multirow{3}{*}{ Tumores } & Sí & 7 & 36.8 & 9 & 47.4 & 3 & 15.8 & 19 \\
\hline & No & 128 & 54.2 & 87 & 36.9 & 21 & 8.9 & 236 \\
\hline & Total & 135 & 52.9 & 96 & 37.6 & 24 & 9.4 & 255 \\
\hline \multirow{3}{*}{ Enfermedad Pulmonar } & Sí & 21 & 42 & 22 & 44 & 7 & 14 & 50 \\
\hline & No & 114 & 55.6 & 74 & 36.1 & 17 & 8.3 & 205 \\
\hline & Total & 135 & 52.9 & 96 & 37.6 & 24 & 9.4 & 255 \\
\hline \multirow{3}{*}{ Problemas del corazón } & Sí & 23 & 62.2 & 12 & 32.4 & 2 & 5.4 & 37 \\
\hline & No & 112 & 51.4 & 84 & 38.5 & 22 & 10.1 & 218 \\
\hline & Total & 135 & 52.9 & 96 & 37.6 & 24 & 9.4 & 255 \\
\hline \multirow{3}{*}{ Problemas de Articulaciones } & Sí & 45 & 51.1 & 32 & 36.4 & 11 & 12.5 & 88 \\
\hline & No & 90 & 53.9 & 64 & 38.3 & 13 & 7.8 & 167 \\
\hline & Total & 135 & 52.9 & 96 & 37.6 & 24 & 9.4 & 255 \\
\hline \multirow{3}{*}{ Problemas de los huesos } & Sí & 39 & 46.4 & 33 & 39.3 & 12 & 14.3 & 84 \\
\hline & No & 96 & 56.1 & 63 & 36.8 & 12 & 7 & 171 \\
\hline & Total & 135 & 52.9 & 96 & 37.6 & 24 & 9.4 & 255 \\
\hline \multirow{3}{*}{ Problemas nerviosos } & Sí & 21 & 37.5 & 28 & 50 & 7 & 12.5 & 56 \\
\hline & No & 114 & 57.3 & 68 & 34.2 & 17 & 8.5 & 199 \\
\hline & Total & 135 & 52.9 & 96 & 37.6 & 24 & 9.4 & 255 \\
\hline \multirow{6}{*}{ Percepción del estado de salud } & Excelente & 13 & 86.7 & 2 & 13.3 & 0 & 0 & 15 \\
\hline & Muy buena & 19 & 63.3 & 11 & 36.7 & 0 & 0 & 30 \\
\hline & Buena & 86 & 65.6 & 41 & 31.3 & 4 & 3.1 & 131 \\
\hline & Regular & 28 & 35 & 39 & 48.8 & 13 & 16.3 & 80 \\
\hline & Mala & 4 & 20 & 9 & 45 & 7 & 35 & 20 \\
\hline & Total & 150 & 54.3 & 102 & 37 & 24 & 8.7 & 276 \\
\hline \multirow{4}{*}{ Autonomía para actividades cotidianas } & Dependiente & 10 & 30.3 & 12 & 36.4 & 11 & 33.3 & 33 \\
\hline & Parcialmente dependiente & 18 & 38.3 & 24 & 51.1 & 5 & 10.6 & 47 \\
\hline & Independiente & 122 & 62.2 & 66 & 33.7 & 8 & 4.1 & 196 \\
\hline & Total & 150 & 54.3 & 102 & 37 & 24 & 8.7 & 276 \\
\hline \multirow{3}{*}{ Presencia de estrés o enfermedad aguda } & Sí & 25 & 35.2 & 32 & 45.1 & 14 & 19.7 & 71 \\
\hline & No & 125 & 61 & 70 & 34.1 & 10 & 4.9 & 205 \\
\hline & Total & 150 & 54.3 & 102 & 37 & 24 & 8.7 & 276 \\
\hline \multirow{3}{*}{ Estado nutricional } & Posible malnutrición & 73 & 48.7 & 58 & 38.7 & 19 & 12.7 & 150 \\
\hline & Normal & 77 & 61.1 & 44 & 34.9 & 5 & 4 & 126 \\
\hline & Total & 150 & 54.3 & 102 & 37 & 24 & 8.7 & 276 \\
\hline
\end{tabular}

Fuente: elaboración propia. 
TABLA 4

Razones de disparidad (OR) para la presencia de sintomas depresivos en los adultos mayores institucionalizados

\begin{tabular}{|c|c|c|c|c|c|}
\hline \multirow{2}{*}{ Característica } & \multirow{2}{*}{ B } & \multirow{2}{*}{$p$} & \multirow{2}{*}{ OR } & \multicolumn{2}{|c|}{ IC OR 95\% } \\
\hline & & & & Lim. Inf. & Lim. Sup. \\
\hline Hombre & & & 1 & & \\
\hline Mujer & -0.51 & 0.2942 & 0.60 & 0.23 & 1.56 \\
\hline $75-84$ & & & 1 & & \\
\hline $65-74$ & 0.17 & 0.7159 & 1.18 & 0.48 & 2.90 \\
\hline $85-+$ & 0.78 & 0.1251 & 2.18 & 0.81 & 5.88 \\
\hline Sin problemas de salud & & & 1 & & \\
\hline Problemas de salud & 0.86 & 0.0626 & 2.36 & 0.96 & 5.84 \\
\hline No problemas nerviosos & & & 1 & & \\
\hline Problemas nerviosos & 0.45 & 0.3666 & 1.56 & 0.59 & 4.13 \\
\hline Independiente & & & 1 & & \\
\hline Dependiente & -1.65 & 0.0943 & 0.19 & 0.03 & 1.33 \\
\hline Parcialmente dependiente & -1.97 & 0.0253 & 0.14 & 0.02 & 0.78 \\
\hline Sin ansiedad & & & 1 & & \\
\hline Riesgo de ansiedad & 1.01 & 0.0272 & 2.74 & 1.12 & 6.72 \\
\hline Sin deterioro funcional & & & 1 & & \\
\hline Deterioro funcional & 1.04 & 0.0355 & 2.82 & 1.07 & 7.41 \\
\hline Estado nutricional normal & & & 1 & & \\
\hline Posiblemente malnutrido & -0.02 & 0.9668 & 0.98 & 0.39 & 2.45 \\
\hline Sin problemas de estrés & & & 1 & & \\
\hline Problemas de estrés & 0.32 & 0.5182 & 1.38 & 0.52 & 3.65 \\
\hline No sentirse maltratado por familiares & & & 1 & & \\
\hline Sentirse maltratado por familiares & -0.16 & 0.7877 & 0.86 & 0.27 & 2.66 \\
\hline Tuvo ingresos económicos el mes anterior & & & 1 & & \\
\hline No tuvo ingresos económicos el mes anterior & 0.23 & 0.5771 & 1.25 & 0.57 & 2.78 \\
\hline Soltero & & & 1 & & \\
\hline Casado & -0.19 & 0.7807 & 0.82 & 0.21 & 3.23 \\
\hline Viudo & -0.80 & 0.0898 & 0.45 & 0.18 & 1.13 \\
\hline Separado/divorciado & 0.35 & 0.5926 & 1.42 & 0.39 & 5.10 \\
\hline Unión libre & -0.24 & 0.8643 & 0.78 & 0.05 & 12.80 \\
\hline Muy satisfecho con apoyo familiar & & & 1 & & \\
\hline Insatisfecho con apoyo familiar & 0.30 & 0.6028 & 1.35 & 0.43 & 4.20 \\
\hline Satisfecho con apoyo familiar & 0.95 & 0.0651 & 2.58 & 0.94 & 7.05 \\
\hline Secundaria/Técnico/Superior & & & 1 & & \\
\hline Ningún nivel educativo & 1.31 & 0.0316 & 3.70 & 1.12 & 12.18 \\
\hline Nivel educativo primaria & 0.60 & 0.1828 & 1.81 & 0.76 & 4.35 \\
\hline La familia no lo llevó a la institución & & & 1 & & \\
\hline La familia lo llevó a la institución & 1.13 & 0.0961 & 3.09 & 0.82 & 11.64 \\
\hline No fue voluntariamente a la institución & & & 1 & & \\
\hline Fue voluntariamente a la institución & -0.02 & 0.9607 & 0.98 & 0.37 & 2.61 \\
\hline
\end{tabular}




\begin{tabular}{lccccc}
\hline \multicolumn{1}{c}{ Característica } & \multirow{2}{*}{ B } & p & OR & \multicolumn{2}{c}{ IC OR 95\% } \\
\cline { 5 - 7 } & & & & Lim. Inf. & Lim. Sup. \\
\hline El motivo para institucionalizarse no fue la soledad & & & 1 & & \\
El motivo para institucionalizarse fue la soledad & 0.35 & 0.4267 & 1.42 & 0.60 & 3.37 \\
\hline $\begin{array}{l}\text { No padece diabetes } \\
\text { Padece diabetes }\end{array}$ & -0.17 & 0.7449 & 0.84 & 0.30 & 2.37 \\
\hline Puntaje de calidad de vida (WHOQoL-OLD) & -0.09 & 0.0001 & 0.92 & 0.88 & 0.96 \\
\hline Mujer e independiente & & & 1 & & \\
Mujer y dependiente & 1.99 & 0.0982 & 7.30 & 0.69 & 77.00 \\
Mujer y parcialmente dependiente & 3.09 & 0.0045 & 21.89 & 2.60 & 184.31 \\
\hline
\end{tabular}

Fuente: elaboración propia.

ajustando simultáneamente por todas las variables independientes, se encontró que aumenta en aquellos con riesgo de ansiedad, déficit funcional, sin formación académica y mujeres parcialmente dependientes. Mientras que la característica que mostró disminuir dicho riesgo fue el puntaje de calidad de vida, donde por cada punto que aumentaba la calidad de vida, la probabilidad de síntomas depresivos se redujo en un $8 \%$. Las demás características no mostraron asociaciones significativas (Tabla 4).

Adicionalmente, fue llevado a cabo un análisis de clases latentes; para ello, se estimaron cinco modelos, donde el modelo con una clase puede ser visto como el de contraste o modelo de línea de base, luego fueron evaluados modelos donde se incrementaron de uno en uno el número de clases latentes. Las medidas de bondad de ajuste se muestran en la Tabla 5, en ella se observa que de los cinco modelos desarrollados, el de mejor ajuste fue el modelo con dos clases.
Tomando en cuenta las características de la población, las dos clases pueden denominarse "buenas condiciones de vida" (clase 1) y "condiciones de vida disminuidas" (clase 2). En la primera clase se incorporó el 36.2\% de la población de adultos mayores, la cual se caracterizó por contar con adultos sin mayores síntomas depresivos; mujeres; adultos de 75 a 84 años de edad; con un nivel educativo secundaria/técnico/superior; pensionados; muy satisfechos con el apoyo familiar, de amigos e institucional; asimismo, quienes se consideraron con un estado de salud bueno y buena percepción de su calidad de vida. Mientras que en la segunda clase, se agrupó el 63.8\% de la población, en los cuales se encontraron aquellos con sintomatología depresiva moderada; mujeres; adultos de 75 a 84 años de edad; con un nivel de formación de primaria; medianamente satisfechos con el apoyo recibido por familiares, amigos e institución; además de aquellos que consideraron como regular su estado de salud y que consideraron como normal su calidad de vida (Tabla 6).

\section{TABLA 5}

Medidas de bondad de ajuste para los cinco modelos de clases latentes aplicados

\begin{tabular}{ccccc}
\hline Clases latentes & Parámetros & LL & Valor $p$ & BIC \\
\hline 1 & 26 & -2857.224 & 0.0000 & 5844.444 \\
2 & 53 & -2713.608 & 0.0000 & 5712.594 \\
3 & 80 & -2667.301 & 0.0000 & 5765.963 \\
4 & 107 & -2639.843 & 0.0000 & 5855.828 \\
5 & 134 & -2614.333 & 0.0000 & 5950.189 \\
\hline
\end{tabular}

Nota. Parámetros: Número de parámetros; LL: Razón de máxima verosimilitud; BIC: Criterio bayesiano de información. Fuente: elaboración propia. 
TABLA 6

Clases latentes observadas y probabilidades condicionales

\begin{tabular}{|c|c|c|c|}
\hline \multicolumn{2}{|l|}{ Característica } & $\begin{array}{c}\text { Clase 1 } \\
(36.24 \%) \\
\end{array}$ & $\begin{array}{c}\text { Clase } 2 \\
(63.76 \%) \\
\end{array}$ \\
\hline \multirow{3}{*}{ Síntomas depresivos } & Normal & 0.7538 & 0.3683 \\
\hline & Depresión Moderada & 0.2462 & 0.4696 \\
\hline & Depresión Severa & 0.0000 & 0.1621 \\
\hline \multirow{2}{*}{ Sexo } & Hombre & 0.3657 & 0.4267 \\
\hline & Mujer & 0.6343 & 0.5733 \\
\hline \multirow{3}{*}{ Edad } & $65-74$ & 0.2373 & 0.3645 \\
\hline & $75-84$ & 0.4799 & 0.3722 \\
\hline & $85-+$ & 0.2828 & 0.2633 \\
\hline \multirow{3}{*}{ Nivel educativo } & Ninguno & 0.084 & 0.2069 \\
\hline & Primaria & 0.438 & 0.5228 \\
\hline & Secundaria/Técnico/Superior & 0.478 & 0.2702 \\
\hline \multirow{2}{*}{ Soledad como motivo de la institucionalización } & No & 0.5823 & 0.7374 \\
\hline & Sí & 0.4177 & 0.2626 \\
\hline \multirow{2}{*}{ La familia lo llevo a la institución } & No & 0.9793 & 0.8652 \\
\hline & Sí & 0.0207 & 0.1348 \\
\hline \multirow{2}{*}{ Fue voluntariamente a la institución } & No & 0.6428 & 0.826 \\
\hline & Sí & 0.3572 & 0.174 \\
\hline \multirow{2}{*}{ Condición en la institución } & Pensionado & 0.8974 & 0.4949 \\
\hline & Caridad & 0.1026 & 0.5051 \\
\hline \multirow{3}{*}{ Satisfacción con apoyo familiar } & Insatisfecho(a) & 0.1039 & 0.3643 \\
\hline & Satisfecho(a) & 0.1293 & 0.6068 \\
\hline & Muy satisfecho(a) & 0.7669 & 0.0289 \\
\hline \multirow{3}{*}{ Satisfacción con apoyo de los amigos } & Insatisfecho(a) & 0.2254 & 0.4969 \\
\hline & Satisfecho(a) & 0.2639 & 0.5031 \\
\hline & Muy satisfecho(a) & 0.5107 & 0.0000 \\
\hline \multirow{3}{*}{ Satisfacción con apoyo de la institución } & Insatisfecho(a) & 0.0431 & 0.1654 \\
\hline & Satisfecho(a) & 0.173 & 0.5139 \\
\hline & Muy satisfecho(a) & 0.7839 & 0.3206 \\
\hline \multirow{2}{*}{ Presencia de problemas de diabetes } & No & 0.7709 & 0.8442 \\
\hline & Sí & 0.2291 & 0.1558 \\
\hline \multirow{4}{*}{ Percepción del estado de Salud } & Muy buena & 0.2309 & 0.0885 \\
\hline & Buena & 0.5701 & 0.3838 \\
\hline & Regular & 0.1797 & 0.4215 \\
\hline & Mala & 0.0192 & 0.1062 \\
\hline \multirow{3}{*}{$\begin{array}{l}\text { Autonomía para desarrollar actividades } \\
\text { cotidianas }\end{array}$} & Dependiente & 0.0264 & 0.2124 \\
\hline & Parcialmente dependiente & 0.229 & 0.1338 \\
\hline & Independiente & 0.7446 & 0.6538 \\
\hline \multirow{3}{*}{ Percepción de la calidad de vida } & Mala & 0.0586 & 0.1486 \\
\hline & Normal & 0.1804 & 0.6494 \\
\hline & Buena & 0.761 & 0.2019 \\
\hline
\end{tabular}

Fuente: elaboración propia. 


\section{Discusión}

Aspectos como baja puntuación en la calidad de vida, no tener alguna formación académica, presentar un deterioro funcional, ser mujer y presentar dependencia para la realización de las actividades cotidianas, así como la comorbilidad con procesos de ansiedad se observaron asociados con mayor posibilidad de presencia de síntomas depresivos por parte del adulto mayor institucionalizado.

El mismo proceso de institucionalización ha sido documentado por algunos autores como desencadenante de procesos depresivos (National Institutes of Health, 1994). Así, algunos autores plantean como la institucionalización en residencias se asocia a factores que favorecen la aparición de cuadros o síntomas depresivos y que pueden dar lugar a un aumento de la prevalencia de este proceso (Cerquera, 2008; Monforte et al., 1998), situación que fue hallada en el presente estudio donde una alta proporción de adultos mayores que fueron llevados por los familiares y/o que no se institucionalizaron de forma voluntaria presentaron síntomas depresivos.

Aunque este proceso de institucionalización resulta ser un claro factor de riesgo de síntomas depresivos en el primer o segundo año de estancia, se ha encontrado que periodos más largos de estadía en el hogar geriátrico se asocian con menos síntomas depresivos (Djernes, 2006), situación que se ve favorecida por la formación de redes sociales al interior de la institución.

La prevalencia de síntomas depresivos hallada en este estudio fue similar a la encontrada por Campos, Ardanaz y Navarro (2004), en su estudio realizado en España, en especial en lo concerniente a la prevalencia de síntomas depresivos moderados. Pero se halló muy superior a lo reportado por otros autores en España (García \& Tobías, 2001; Urbina et al., 2007), Pereira (García et al., 2003) y Taguatinga-Brasilia (Oliveira, Gomes \& Oliveira, 2006).

Las causas que llevan a un sujeto a deprimirse e incluso a trastornarse son múltiples, sin existir aún un consenso por parte de los investigadores; dentro de estos factores se hallan la viudez, relaciones sociales, apoyo familiar y circunstancias demográficas (Hernández, 2001). Con relación a la viudez y con- trario a lo planteado de forma inicial y a lo hallado por Ibarzábal (2003) en su estudio en adultos mayores institucionalizados y no institucionalizados, esta investigación no encontró asociación entre el estado marital del adulto mayor y la sintomatología depresiva.

En lo que respecta al sexo del adulto mayor, este resulta ser divergente en cuanto a si las mujeres son más proclives a presentar síntomas depresivos o no, pues a pesar de que son varios los autores que han reportado mayores prevalencias de síntomas depresivos en ellas (Djernes, 2006; García et al., 2001; Gómez-Restrepo et al., 2004; Márquez, Soriano, García \& Falcón, 2005; Vink, Aartsen \& Schoevers, 2008), otros estudios y esta investigación no han encontrado tales diferencias por sexo (Acosta \& García, 2007; Ávila-Funes et al., 2006; Campos et al., 2004; Urbina et al., 2007). Estas divergencias en los estudios con relación al sexo, pudieran tener una explicación debido a las variaciones en arreglos socioculturales de las poblaciones. Aunque también pudiera deberse al grupo de población específico al cual se haga referencia, puesto que esta mayor proporción de mujeres depresivas es más notoria entre los 15 y los 70 años. En los otros grupos de edad existe un equilibrio a este respecto entre los dos géneros (Alonso-Fernández, 2001; Del Moral, Puigdemont, Serrano \& Pérez, 1995).

Algunos de los factores de riesgo psicológicos para la presencia de trastornos depresivos durante la tercera edad y que determinan el proceso del envejecimiento son: una posición de total dependencia (Zarebski, 1999 citado en Hernández, 2001), alteraciones del funcionamiento cognitivo (Ávila-Funes et al., 2006; Ibarzábal, 2003) y una coexistencia elevada con ansiedad (Martínez-Mendoza, Martínez-Ordaz, Esquivel-Molina \& Velasco-Rodríguez, 2007), siendo reportada por algunos autores dicha coexistencia hasta de un 60\% (Heinze et al., 1999), otros afirman también que el 50\% de las ansiedades generalizadas tienen síntomas de depresión (Alonso-Fernández, 2001; Conde \& Jarne, 2006). Esta situación se correlacionó con los hallazgos encontrados en el presente estudio, el cual halló que de las personas con riesgo de ansiedad, el 78\% presentaba algún grado de sintomatología depresiva. 
La presencia de alguno de estos trastornos o de ellos en su conjunto causan deterioro del funcionamiento orgánico y psicoemocional, perdida de la independencia y de la autonomía, alta utilización de los servicios asistenciales, aumentando la mortalidad por comorbilidades (Blazer, Hybels \& Pieper, 2001).

En lo que respecta a la autonomía del adulto mayor, al igual que lo hallado por algunos autores (Ávila-Funes et al., 2006; Campos et al., 2004; Urbina et al., 2007), este estudio encontró que la mayor prevalencia de síntomas depresivos estuvo asociada con la dependencia por parte del adulto mayor para realizar las actividades diarias. Situación que parece clara, teniendo en cuenta que la incapacidad y la dependencia implican sentimientos de frustración que generan depresión.

Los adultos mayores que viven con personas no cercanas al núcleo familiar, que cuentan con poco apoyo de sus familias o que no se sienten tan satisfechas con dicho apoyo, tienden a presentar más síntomas de depresión que quienes cuentan con un apoyo familiar más cercano y satisfactorio (Aranda et al., 2001; Aranibar, 2001; Márquez et al., 2005). Esto pudiera deberse a que en la vejez, las personas se encuentran cada vez con más limitaciones físicas, aumentando la importancia de los amigos y familiares para responder a las actividades instrumentales cotidianas, para la alta autoestima y la gratificación positiva que resulta de los lazos interpersonales (Buendía \& Riquelme, 1994).

En cuanto a la calidad de vida del adulto mayor institucionalizado, se ha evidenciado que los que tienen una peor calidad de vida muestran mayores prevalencias de síntomas depresivos (Wada et al., 2005; Xavier et al., 2002), lo que corresponde con lo evidenciado en este estudio. Situación que debe analizarse con sumo cuidado, pues puede estar sesgada por los mismos signos de la depresión, lo que pudo derivar en que esta calificación de calidad de vida se haya subvalorado. A pesar de lo anterior, los resultados sugieren incluso que menores niveles de depresión están asociados con un decremento significativo en la calidad de vida y con un patrón de actitudes negativas hacia esta (Chachamovich et al., 2008).
El presente estudio presentó algunas limitaciones, como fueron la imposibilidad de acceder a todas las instituciones públicas o privadas que albergaban población adulta mayor. Respecto a los adultos mayores encuestados, la limitante encontrada fue que solo pudo hablarse de residentes sin discapacidades mentales que les impidieran responder la encuesta diseñada en el estudio. Pese al proceso de estandarización y capacitación en la toma de medidas corporales, se contaron con algunos pocos casos en los que los adultos mayores no quisieron quitarse sus zapatos y otros no permitieron ser despojados de algunas de sus prendas, lo que pudo introducir algún sesgo en estas medidas.

La presencia de sintomatología depresiva mostró deterioro de la calidad de vida de los adultos mayores institucionalizados, pues la pérdida de roles, de seres queridos y el sentirse en un estado de dependencia, los hacen más vulnerables. Estos resultados evidenciaron que la sintomatología depresiva es bastante común en adultos mayores institucionalizados, requiriendo el establecimiento de estrategias preventivas.

\section{Referencias}

Acosta, C. O. \& García, R. (2007). Ansiedad y depresión en adultos mayores. Psicología y Salud, 17(2), 291-300.

Agresti, A. (2002). Categorical data analysis (2.a ed.). New York: Wiley Interscience.

Alarcón, R. P. \& García, L. H. (2003). Trastornos depresivos en poblaciones ancianas en el departamento de Risaralda. Tipos de trastornos y factores de riesgo individuales y socio-familiares. Revista de la Asociación Colombiana de Gerontología y Geriatría, 17(3), 506-517.

Alexandre, T. S., Cordeiro, R. C. \& Ramos L. R. (2009). Factors associated to quality of life in active elderly. Revista de Saúde Pública, 43(4), 613-621.

Alonso-Fernández, F. (2001). Claves de la depresión. Madrid: Cooperación Editorial.

Aranda, C., Pando, M., Flores, M. E. \& García, T. (2001). Depresión y redes sociales de apoyo en el adulto mayor institucionalizado de la zona metropolitana de Guadalajara, Jalisco. Revista de 
Psiquiatría de la Facultad de Medicina de Barcelona, 28(2), 69-74.

Aranibar, P. (2001). Acercamiento conceptual a la situación del adulto mayor en América Latina (Serie Población y Desarrollo No. 21). Santiago de Chile: CEPAL/CELADE.

Ávila-Funes, J. A., Garant, M. P. \& Aguilar-Navarro, S. (2006). Relación entre los factores que determinan los síntomas depresivos y los hábitos alimentarios en adultos mayores de México. Revista Panamericana de Salud Pública, 19(5), 321-330.

Bacca, A. M., González, A. \& Uribe, A. F. (2005). Validación de la escala de depresión de Yesavage (Versión reducida) en adultos mayores colombianos. Pensamiento Psicológico, 1(4), 53-63.

Blazer, D. G., Hybels, C. F. \& Pieper, C. F. (2001). The association of depression and mortality in elderly persons: A case for multiple, independent pathways. Journals of Gerontology Series A: Biological Sciences and Medical Sciences, 56(8), M505-509.

Buendía, J. \& Riquelme, A. (1994). Envejecimiento y depresión. En J. Buendía (Comp.), Envejecimiento y psicología de la salud (pp. 219-233). Madrid: Siglo XXI.

Campos, J., Ardanaz, J. \& Navarro, A. (2004). Depresión en pacientes de edad avanzada. Dos ámbitos: un centro sociosanitario y un programa de soporte domiciliario. Revista Española de Geriatría y Gerontología, 39(4), 232-239.

Cerquera, A. M. (2008). Relación entre los procesos cognitivos y el nivel de depresión en las adultas mayores institucionalizadas en el asilo San Antonio de Bucaramanga. Universitas Psychologica, 7(1), 271-281.

Chachamovich, E., Fleck, M., Laidlaw, K. \& Power, M. (2008). Impact of major depression and subsyndromal symptoms on quality of life and attitudes toward aging in an international sample of older adults. The Gerontologist, 48(5), 593-602.

Colombia, Departamento Administrativo Nacional de Estadística. (2007). Censo general 2005: población adulta mayor. Bogotá: Autor. Disponible en http:// dane.gov.co

Conde, J. L. \& Jarne, A. (2006). Trastornos mentales en la vejez. En C.Triado \& F. Villar (Comps.), Psicología de la vejez (pp. 341-364). Madrid: Alianza.
Del Moral, E. J., Puigdemont, D., Serrano, D. \& Pérez, J. (1995). Depresión en el anciano. Epidemiología, clínica y diagnóstico. Jano, 49, 871-876.

Djernes, J. K. (2006). Prevalence and predictors of depression in population of elderly: A review. Acta Psychiatrica Scandinavica, 113(5), 372-387.

García, M. J. \& Tobías, J. (2001). Prevalencia de depresión en mayores de 65 años. Perfil del anciano de riesgo. Atención Primaria, 27(7), 484-488.

Goldberg, D., Bridges, K., Duncan-Jones, P. \& Grayson, D. (1988). Detecting anxiety and depression in general medical settings. British Medical Journal, 297(6653), 897-899.

Gómez-Restrepo, C., Bohórquez, A., Pinto, D., Gil, J. F. A., Rondón, M. \& Díaz-Granados, N. (2004). Prevalencia de depresión y factores asociados con ella en la población colombiana. Revista Panamericana de Salud Pública, 16(6), 378-386.

Guigoz, Y., Vellas, B. \& Garry, P. J. (1994). The Mini Nutritional Assessment (MNA): A practical assessment tool for grading the nutritional state of elderly patients. En Y. Guigoz, B. Vellas, P. J. Garry \& J. L. Albarede (Eds.), Facts and Research in Gerontology (Suppl. 2, pp. 15-59). New York: Springer.

Heinze, G., Escalante, C. A., Fernández, R., Galli, E., León, C. A., Moreno, R. A., et al. (1999). The coexistence of depression and anxiety. Salud Mental, 22(3), 820-826.

Hernández, Z. E. (2001). ¿Por qué se deprimen los viejos? Revista Intercontinental de Psicología y Educación, 3(2), 31-38.

Hirschfeld, M. \& Lindsey, E. (2002). Community homebased care in resource-limited settings: A framework for action. Geneva: World Health Organisation.

Ibarzábal, A. (2003). Personas mayores institucionalizadas versus no institucionalizadas: aspectos diferenciales en ansiedad ante la muerte, depresión y satisfacción con la vida. MAPFRE Medicina, 14(4), 258-264.

Jackson, A. S. \& Pollock, M. L. (1985). Practical assessment of body composition. The Physician and Sportsmedicine, 13(5), 76-89.

Lebowitz, B. D., Pearson, J. L., Schneider, L. S., Reynolds, C. F., 3rd, Alexopoulos, G. S., Bruce, M. L., et al. (1997). Diagnosis and treatment of depression 
in late life. Consensus statement update. JAMA, 278(14), 1186-1190.

Márquez E., Soriano S., García A. \& Falcón, M. P. (2005). Depresión en el adulto mayor: frecuencia y factores de riesgo asociados. Atención Primaria, 36(6), 125-126.

Martínez-Mendoza, J. A., Martínez-Ordaz, V. A., Esquivel-Molina, C. G. \& Velasco-Rodríguez, V. M. (2007). Prevalencia de depresión y factores de riesgo en el adulto mayor hospitalizado. Revista Médica. Instituto Mexicano del Seguro Social, 45(1), 21-28.

Minor, L. \& Kaemppffmam, G. (2006, marzo). La problemática del anciano institucionalizado. Geronto-geriatría. Órgano oficial de la Federación Argentina de Geriatría y Gerontología [Online]. Recuperado de http://www.gerontogeriatria.org.ar/pdf/la_problematica_del_anciano_institucionalizado.pdf

Monforte, J. A., Fernández, C., Díez, J., Toranzo, I., Alonso, M. M. \& Franco, M. A. (1998). Prevalencia de la depresión del anciano que vive en residencias. Revista Española de Geriatría y Gerontología, 33(1), 13-20.

National Institutes of Health. (1994). Diagnosis and treatment of depression in late life: Results of the NIH Consensus Development Conference (1991: Nov. 4-6). Washington: American Psychiatric Press, Inc.

Oliveira, D. A., Gomes, L. \& Oliveira, R. F. (2006). Prevalence of depression among the elderly population who frequent community centers. Revista de Saúde Pública, 40(4), 734-736.

Organización de las Naciones Unidas. (2009). Proyección de población (Observatorio Demográfico Año 4№ 7. América Latina y el Caribe). Santiago de Chile: CEPAL/UNFPA.

Organización Mundial de la Salud. (2006). Manual WHOQOL-OLD. Brazil. Recuperado el 1 de no- viembre de 2009, de http://www.ufrgs.br/psiq/escalas.html

Pérez, V. T. \& Arcia, N. (2008). Comportamiento de los factores biosociales en la depresión del adulto mayor [Versión electrónica]. Revista Cubana de Medicina General Integral, 24(3).

Santos, S. S. C., Tier, C. G., Silva, B. T, Barlem, E. L. D., Felicianni, A. M. \& Valcarenghi, F. V. (2010). Diagnósticos e intervenciones de enfermería para ancianos con depresión y residentes en una institución de larga estancia (ILE) [Versión electrónica]. Enfermería Global, 9(20).

Urbina, J. R., Flores, J. M., García, M. P., Torres, L. \& Torrubias, R. M. (2007). Síntomas depresivos en personas mayores. Prevalencia y factores asociados. Gaceta Sanitaria, 21(1), 37-42.

Vink, D., Aartsen, M. \& Schoevers, R. (2008). Risk factors for anxiety and depression in the elderly: A review. Journal of Affective Disorders, 106(1-2), 29-44.

Wada, T., Ishine, M., Sakagami, T., Kita, T., Okumiya, K., Mizuno, K., et al. (2005). Depression, activities of daily living, and quality of life of communitydwelling elderly in three Asian countries: Indonesia, Vietnam, and Japan. Archives of Gerontology and Geriatrics, 41(3), 271-280.

Xavier, F., Ferraza, M. P., Argimon, I., Trentini, C. M., Poyares, D., Bertolluci, P. H., et al. (2002). The DSM-IV 'minor depression' disorder in the oldest-old: Prevalence rate, sleep patterns, memory function and quality of life in elderly people of Italian descent in Southern Brazil. International Journal of Geriatric Psychiatry, 17(2), 107-116.

Yesavage, J. A., Brink, J. 1., Rose, T. L., Lum, O., Adey, M. \& Leirer, V. O. (1982). Development and validation of a geriatric depression screening scale: $\mathrm{A}$ preliminary report. Journal of Psychiatric Research, 17(1), 37-49. 